\title{
APPROXIMATION BY INVERTIBLES
}

\author{
MICHAEL GARTENBERG
}

\begin{abstract}
The uniform closure of the set of invertibles of certain $C^{*}$ algebras is characterized. It is shown that indices on $\mathcal{G}(C(A))$, where $A$ is a closed annulus in the plane and on $M_{2}^{S^{2} \times S^{1}}$ have continuous extensions to elements which are not invertible.
\end{abstract}

1. Introduction. It is well known that the set of invertible elements $\mathcal{G}$ in a Banach algebra is open in the algebra. Feldman and Kadison have studied the uniform closure of $\mathcal{G}$ where the algebra in question is $9 \mathcal{B}(\mathcal{H})$ [6]. Concerning $C^{*}$-algebras, in general, less is known, and we discuss this problem for certain function algebras.

A related problem is one which can be phrased very generally. Let $\mathcal{Q}$ be a $C^{*}$-algebra with identity, and let $\mathcal{G}$ denote the set of invertibles in $\mathcal{Q}$. A continuous homomorphism $i$ of $\mathcal{G}$ into some discrete group $\mathscr{D}$ is called an index on $\mathcal{G}$. If $r \in \mathcal{D}$, we write $\mathcal{G}_{r}=i^{-1}(r)$. For any given index we can define the set

$$
\begin{aligned}
\mathscr{H} C=\{x \in \overline{\mathcal{G}}: \text { there exists an } \varepsilon>0 \\
\left.\quad \text { and a fixed } r(x) \in \mathcal{D}: N_{\varepsilon(x)}(x) \cap \mathcal{G} \subset \mathcal{G}_{r(x)}\right\},
\end{aligned}
$$

and for $r(x) \in \mathscr{D}$ we write $\mathscr{H}_{r}=\{x \in \mathscr{H}: r(x)=r\}$.

We consider the problem of determining $\mathscr{H}$ for certain $C^{*}$-algebras whose elements are symbols of operators on a Hilbert space. Coburn and Lebow have considered the case of the usual index defined on the invertibles of the Calkin algebra $\mathscr{B}(\mathcal{H}) / \mathscr{K}$ where $\mathscr{H}$ is a separable Hilbert space and $\mathscr{K}$ is the set of compact operators on $\mathcal{H}$. Here $\mathscr{H}=\stackrel{?}{3}[3]$.

The following is an immediate result of the definition.

Proposition 1. (i) If $i^{\prime}$ is defined on $\mathcal{H}$ by $i^{\prime}(x)=r(x)$, then $i^{\prime}: \mathcal{H} \rightarrow$ OD is continuous, and $i^{\prime}$ restricted to $\mathcal{G}$ equals $i$. Each $\mathcal{H}_{r}$ is open in $\mathcal{H}$.

(ii) $\mathcal{H}$ is not necessarily closed under products, but if $x \in \mathcal{H}_{r}, y \in \mathscr{H}_{s}$ and $x y \in \mathcal{H}$, then $x y \in \mathcal{H}_{r+s}$.

(iii) $\mathcal{G}_{r} \mathcal{H}_{s} \subset \mathcal{H}_{r+s}$.

(iv) $\mathcal{G}_{r}\left(\mathcal{H}_{s}-\mathcal{G}_{s}\right) \subset \mathcal{H}_{r+s}-\mathcal{G}_{r+s}$.

2. The algebra $Q=C(T)$. Let $Q=C(T)$ be the algebra of all complexvalued continuous functions on the unit circle $T$. A function $f \in Q$ is invertible if and only if $f$ does not vanish on $T$. The winding number of the

Received by the editors January 22, 1974 and, in revised form, July 8, 1974 and April 2, 1975. AMS (MOS) subject classifications (1970). Primary 47B05; Secondary 47B30.

Key words and phrases. $C^{*}$-algebras, extensions of indices, invertible element. 
curve traced out by $f \in \mathcal{G}(\mathbb{Q})$ with respect to origin, denoted by w.n. $(f)$ determines an index on $\mathcal{G}$ [2].

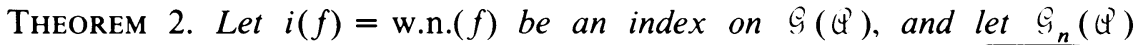
$=\{f \in \mathcal{G}(\mathbb{Q}): i(f)=n\}$. Then for any $f \in \mathbb{Q}-\mathcal{G}(\mathbb{Q}), f \in \cap_{n} \overline{\mathcal{G}_{n}(\mathbb{Q})}$, so that $\mathfrak{H}=\mathcal{G}$.

Proof. Let $\varepsilon>0$ be given, and set $S_{\varepsilon}=\{z \in T:|f(z)|<\varepsilon\}$. Consider the function $\phi \in C(T)$ defined as follows. For $z \in T-S_{\varepsilon}, \phi(z)=f(z)$; for $z=e^{i \omega} \in I_{i}$ where $I_{i}=\left\{e^{i \omega}: \omega_{0}<\omega<\omega_{1}\right\}$ is an arbitrary interval of $S_{\varepsilon}$ for which $f\left(e^{i \omega_{0}}\right)=\varepsilon e^{i \omega_{0}}$ and $f\left(e^{i \theta_{1}}\right)=\varepsilon e^{i \theta_{1}}$, let

$$
\phi\left(e^{i \omega}\right)=\varepsilon \exp \left\{i\left[\left(\frac{\omega_{1}-\omega_{0}}{\theta_{1}-\theta_{0}}\right)\left(\omega-\omega_{0}\right)+\theta_{0}\right]\right\} .
$$

The function $\phi \in \mathcal{G}(\mathbb{Q})$, and $\|f-\phi\| \leqslant 2 \varepsilon$ on $T$. Since $\varepsilon>0$ is arbitrary, $f \in \overline{\mathcal{G}(\mathbb{Q})}$.

The function $\phi$ can have arbitrary index. For if $i(\phi)=n$ and $k$ is any integer other than $n$, the function $\phi^{\prime} \in \mathcal{G}\left(\mathcal{G}^{\prime}\right)$ defined by $\phi^{\prime}(z)=\phi(z)$ for $z \in T-I_{1}$ where $I_{1}=\left\{e^{i \omega}: \omega_{0}<\omega<\omega_{1}\right\}$ is some fixed interval of $S_{\varepsilon}$, and by

$$
\phi^{\prime}(z)=\left\{\begin{array}{c}
\varepsilon \exp \left\{z i\left[\frac{\theta_{1}-\theta_{0}}{\omega_{1}-\omega_{0}}\right]\left(\omega-\omega_{0}\right)+i \theta_{0}\right\}, \\
\omega_{0}<\omega<\frac{\omega_{0}+\omega_{1}}{2}, \\
\varepsilon \exp i \omega_{1} \exp \left\{\frac{-4 \pi i(k-n)}{\omega_{1}-\omega_{0}}\left(\omega-\left(\frac{\omega_{0}-\omega_{1}}{2}\right)\right)\right\}, \\
\frac{\omega_{0}+\omega_{1}}{2}<\omega<\omega_{1},
\end{array}\right.
$$

has index $k$, and $\left\|f-\phi^{\prime}\right\|<2 \varepsilon$ on $T$. Since $\varepsilon>0$ and $k$ are arbitrary, we conclude that $f \in \cap_{n} \overline{\mathcal{G}_{n}(\mathbb{Q})}$.

Since every neighborhood of $f$ contains invertibles of all indices, $\mathcal{H}=\mathcal{G}$.

We next recall some basic notions of Fredholm theory. An operator $A$ in $\mathscr{B}(\mathcal{H})$ is said to be Fredholm if the range of $A$ is closed and $\operatorname{dim} \operatorname{ker} A+$ $\operatorname{dim} \operatorname{ker} A^{*}$ is finite. Atkinson has shown that these conditions are equivalent to $\pi(A)$ being invertible in the algebra $93(\mathcal{H}) / \mathscr{K}$ where $\pi$ is the quotient map [1]. The analytic index $i_{\text {an }}$ defined on the set of Fredholm operators $\mathscr{F}$ by $i_{\text {an }}(A)=\operatorname{dim} \operatorname{ker} A-\operatorname{dim} \operatorname{ker} A^{*}$ is a homomorphism of $\mathscr{F}$ onto the integers.

Now for $\phi \in C(T)$, the Toeplitz operator $T_{\phi}$ is defined as follows: Let $L^{2}(T)$ be the space of Lebesgue square-integrable functions on $T$ with the orthonormal basis $\left\{e^{i n x}, n \in Z\right\}$. The subspace spanned by those $e^{i n x}$ for which $n \geqslant 0$ is denoted by $H^{2}(T)$. The operator $T_{\phi}$ is defined on $H^{2}(T)$ by $T_{\phi} f=P(\phi f)$ where $P$ is the orthogonal projection from $L^{2}$ onto $H^{2}$.

The Banach algebra $\mathscr{T}$ generated by $T_{z}$ has been studied by Coburn [2]. He has shown that $B=\left\{T_{\phi}+K: \phi \in C(T), K\right.$ compact $\}$ and that the $C^{*}$-algebra $\Re / \mathcal{K}$ is isometrically ${ }^{*}$-isomorphic to $C(T)$. It follows that $T_{\phi}$ is 
a Fredholm operator if and only if $\phi$ does not vanish on $T$ and that $i_{\text {an }}\left(T_{\phi}\right)$ equals the negative of w.n. $(\phi)$ with respect to the origin. Lastly, let the map $\rho$ : $\Re \rightarrow C(T)$ be given by $\rho\left(T_{\phi}+K\right)=\phi$. It has been shown that the natural cross section $s$ of $\rho$ is continuous. Thus for any component $\mathcal{C}$ of $\mathcal{G}(C(T))$, $s(\bar{C})=\overline{s(C)}[7]$.

These remarks together with Theorem 2 yield the following analogue of Theorem 2 for the set of Fredholm operators $\mathscr{F}$ in $\mathscr{B}$.

COROLlary 3. There is no possibility of continuously extending the analytic index beyond $\mathscr{F}$.

Let $\Omega$ be a compact set in the plane with interior whose boundary consists of finitely many Jordan curves. If $i$ is the index defined on $\mathcal{G}(C(\Omega))$ by $i(f)=0$, then $\mathcal{H}=\overline{\mathcal{G}}$ is always larger than $\mathcal{G}$. The problem is to determine $\mathcal{G}$. We limit ourselves to the following

THEOREM 4. If $\Omega$ is a set of the type described, then $\mathcal{G} \varsubsetneqq \overline{\mathcal{G}} \varsubsetneqq C(\Omega)$.

Proof. Let $a$ be an interior point of $\Omega$. The function $f(z)=z-a$ in $C(\Omega)$ has nonzero winding number along closed curves in $\Omega$ about the point $a$. Hence $f$ cannot be uniformly approximated by nonvanishing functions of $C(\Omega)$.

On the other hand, if $f \in C(\Omega)$ vanishes only on $\partial \Omega$, then $f \in \overline{\mathcal{G}}$. For any component $\circlearrowright$ of $\Omega$ with interior and any $\varepsilon>0$, let $I=\{z \in \partial \circlearrowright:|f(z)|$ $<\varepsilon / 2\}$. Then $I=\cup_{i} I_{i}$ is a countable union of disjoint smooth curves. There exist smooth $\operatorname{arcs} \gamma_{i}$ in $\mathcal{C}$ having as endpoints the endpoints of $\bar{I}_{i}$ such that on the region $R_{i}$ bounded by $\bar{I}_{i}$ and $\gamma_{i},|f(z)|<\varepsilon$. On each $R_{i}$ there is a continuous function $f_{i}$ satisfying $f_{i \mid \gamma_{i}}=f_{\mid \gamma_{i}}$ with the range of $f_{i}$ on all of $R_{i}$ equal to the range of $f$ on $\gamma_{i}$. The function $f^{\prime}$ defined by

$$
f^{\prime}(z)= \begin{cases}f(z), & z \in \mathcal{C}-\bigcup_{i} R_{i}, \\ f_{i}(z), & z \in R_{i},\end{cases}
$$

is continuous and nonvanishing on $\mathcal{E}$, and $\left\|f-f^{\prime}\right\|<2 \varepsilon$ on $己$. Since $\mathcal{E}$ and $\varepsilon>0$ are arbitrary, $f \in \overline{\mathcal{G}}$.

3. The algebra $\mathbb{Q}=C(A)$. We now describe an algebra for which there exists a nontrivial index that can be continuously extended beyond $\mathcal{G}(\mathbb{Q})$. Let $A$ be the closed annulus in the plane centered at the origin with radii $r=\frac{1}{2}$ and $r=1$. A function $f \in \mathbb{Q}=C(A)$ is invertible if and only if $f$ does not vanish on $A$, and the winding number traced out by $f$ with respect to the origin along the curve $r=\frac{3}{4}$ determines an index on ${ }_{i}\left(G^{\prime}\right)$.

Theorem 5. For the index described, $\mathcal{S} \subsetneq \mathcal{H} \subsetneq \overline{\mathcal{G}} \subsetneq C(A)$.

Proof. If $f \in C(A)$ vanishes only on $\partial A$, then by Theorem $4, f \in \overline{\mathcal{G}(Q)}$. The winding number of $f$ along $r=\frac{3}{4}$ is some integer, say $n$. It follows from the continuity of the winding number that if $g \in \mathcal{G}\left(\mathcal{U}^{\prime}\right)$ satisfies $\|f-g\|<\varepsilon$ for $\varepsilon>0$ sufficiently small, then w.n. $(g)$ along $r=\frac{3}{4}$ is also $n$. Hence, $f \in\left(\mathcal{H}_{n}-\mathcal{G}_{n}\right)$. 
On the other hand, let $g \in C(A)$ be defined by $g\left(r e^{i \theta}\right)=g\left(e^{i \theta}\right), \frac{1}{2} \leqslant r$ $\leqslant 1 ; g\left(r e^{i \theta^{\prime}}\right)=0$ for some fixed $\theta^{\prime}$, and $g\left(r e^{i \theta}\right) \neq 0$ for $\theta \neq \theta^{\prime}$. It follows from Theorem 2 that $g \in \cap_{n} \overline{\mathcal{G}_{n}(\mathscr{Q})}$, so that $g \notin \mathcal{H}$. Hence, $\mathcal{H} \subsetneq \overline{\mathcal{G}}$. Finally, it follows from Theorem 4 that if $h \in C(A)$ has an isolated zero at an interior point of $A$, then $h \notin \overline{\mathcal{G}}$.

The elements of $C(A)$ can be realized as symbols of operators on a Hilbert space. Because of this, we can characterize a distinguished class of nonFredholm operators to which the analytic index can be continuously extended.

Let $\mathcal{H}=L^{2}(A, d A) \oplus H^{2}\left(\frac{3}{4} T, d \theta\right)$, where $L^{2}(A, d A)$ is the Hilbert space of all square-integrable functions on $A$ with respect to area measure and $H^{2}\left(\frac{3}{4} T, d \theta\right)$ is the Hardy space of all square-integrable functions on $\frac{3}{4} T$ with respect to normalized Haar measure. For $\phi \in C(A), M_{\phi}$ denotes the multiplication operator on $L^{2}(A, d A)$ induced by $\phi$ and $T_{\tilde{\phi}}$ denotes the Toeplitz operator on $H^{2}\left(\frac{3}{4} T, d \theta\right)$ induced by $\tilde{\phi}=\left.\phi\right|_{\frac{3}{4} T}$. We shall denote by $S_{\phi}$ the operator $M_{\phi} \oplus T_{\tilde{\phi}}$ on $\mathcal{H}$.

Lemma 6. For any $\phi, \psi \in C(A)$ the following properties hold:

(i) $S_{\phi}^{*}=S_{\phi}$;

(ii) $S_{\lambda \phi+\mu \psi}=\lambda S_{\phi}+\mu S_{\psi}$ for any complex scalars $\lambda$ and $\mu$;

(iii) $\left\|S_{\phi}\right\|=\left\|\pi\left(S_{\phi}\right)\right\|=\|\phi\|$.

Proof. (i) and (ii) are direct computations; (iii) is a consequence of the Douglas generalization of the Berg-Weyl theorem [5].

Theorem 7. The set $\Theta=\left\{S_{\phi}+K, K \in \mathcal{K}\left(\mathcal{H}^{\circ}\right)\right\}$ is a Banach algebra, and the representation of elements of $A^{\prime}$ as sums is unique.

Proof. It follows from the lemma that if the operators $A$ and $B$ are in $Q$, then so are $A+B, \lambda \cdot A$, and $A^{*}$. Since $T_{\tilde{\phi}} T_{\tilde{\psi}}-T_{\tilde{\phi} \tilde{\psi}}$ is a compact operator on $H^{2}\left(\frac{3}{4} T, d \theta\right)$ [2], $S_{\phi} S_{\psi}-S_{\phi \psi}$ is in $\mathcal{K}(\mathcal{H})$, and $\mathscr{Q}$ is closed under multiplication. It remains to be shown that the algebra $Q$ is closed. By (iii) above, if a sequence $\left\{S_{\phi_{n}}+K_{n}\right\}$ is Cauchy, then so is $\left\{\phi_{n}\right\}$. But then $\phi_{n} \rightarrow \phi$ for some $\phi \in C(A)$. Again by (iii), $S_{\phi_{n}} \rightarrow S_{\phi}$ so that $K_{n} \rightarrow K$ for some $K \in \mathcal{K}(\mathcal{H})$, and $\mathcal{Q}$ is closed. Lastly, the norm equality $\left\|S_{\phi}+K\right\|=\|\phi\|$ implies that the representation is unique.

Corollary 8. The map $S_{\phi}+K \leftrightarrow \phi$ is an isometric *-isomorphism between $Q / \mathcal{K}$ and $C(A)$. The operator $S_{\phi}$ is Fredholm in ${ }^{\prime}$ if and only if $\phi$ does not vanish on $A$, and the analytic index on $S_{\phi}$ equals minus the winding number of $\phi$ along $r=\frac{3}{4}$ with respect to the origin.

Proof. The first remarks follow immediately from Proposition 6, Theorem 7 , and Atkinson's characterization of Fredholm operators. Since ind $S_{\phi}$ = ind $M_{\phi}+$ ind $T_{\tilde{\phi}}=0+$ w.n.. $(\tilde{\phi})$ [5], we have the index characterization of the corollary.

COROLlary 9. The analytic index on the set of Fredholms $\mathcal{F} \subset$ (i) can be continuously extended to those elements of $\bar{T}-\bar{r}$ whose symbols lies in $\bar{Y}$.

Proof. This is an immediate consequence of Theorem 5 and the fact that the map $\phi \rightarrow S_{\phi}$ is continuous. 
4. The algebra $\mathscr{Q}=M_{2}^{S^{2} \times S^{1}}$. We will consider $S^{2}$ as the one point compactification of the plane and $S^{1}$ the unit sphere $|z|=1$. We shall study the $C^{*}$-algebra $Q=M_{2}^{S^{2} \times S^{1}}$ of $2 \times 2$ matrix-valued functions on $S^{2} \times S^{1}$ in the matrix supremum norm. This algebra can be identified as the symbol space of the algebra $\mathscr{B}$ of $2 \times 2$ systems of singular integral operators. We briefly describe this latter algebra and refer the reader to [4] for details.

Let $\mathscr{F}_{f}$ denote the Fourier transform of the function $f(x) \in L^{2}\left(R^{2}\right)$. The algebra $\mathscr{B}$ is generated by $2 \times 2$ systems of (a) the multiplication operators $M_{\phi}, \phi \in C\left(S^{2}\right)$, (b) $\mathscr{F}^{-1} M_{k(x)} \mathscr{F}$ where $k(x) \in C\left(S^{1}\right)$ is homogeneous of degree zero, and (c) the ideal $\mathcal{K}$ of compact operators on $L^{2}\left(R^{2}\right) \oplus L^{2}\left(R^{2}\right)$. The symbol map $\sigma$ on $\beta$ given by

$$
\begin{aligned}
\sigma\left(M_{\phi}\right) & =\phi(x), \quad x \in S^{2} ; \\
\sigma\left(\mathscr{F}^{-1} M_{k} \mathscr{F}\right) & =k(\theta), \quad \theta \in S^{1} ; \\
\sigma(K) & =0, \quad K \text { compact; }
\end{aligned}
$$

can be extended to a ${ }^{*}$-homomorphism from all of $\mathscr{B}$ onto $M_{2}^{S^{2} \times S^{1}}$ and has a continuous cross section. An operator in $\mathscr{B}$ is Fredholm if and only if its symbol has nonvanishing determinant on $S^{2} \times S^{1}$, and two Fredholm operators are homotopic if and only if their symbols, considered as matrix functions from $S^{2} \times S^{1}$ into $\mathrm{GL}_{2}$, the general linear group of all $2 \times 2$ nonsingular matrices, are homotopic.

An index $i: \mathcal{G}=\mathrm{GL}_{2}^{S^{2} \times S^{1}} \rightarrow Z$ is determined as follows. If for $f \in \mathcal{G}$, we write $f=p u$, where $p$ is positive and

$$
u=\left(\begin{array}{ll}
u_{1} & u_{2} \\
u_{3} & u_{4}
\end{array}\right) \in U_{2}^{S^{2} \times S^{1}},
$$

the $2 \times 2$ unitary matrices in $\mathcal{G}$, then the map $\gamma(u)=\left(u_{1}, u_{2}\right)$ determines an element of $S^{3^{S^{2} \times S^{1}}}$. Now $\pi_{0}\left(S^{3^{S^{2} \times S^{1}}}\right) \simeq Z$, and we define $i(f)=[\gamma \circ u]$ $\in \pi_{0}\left(S^{3^{S^{2} \times S^{\prime}}}\right)$, where $[\gamma \circ u]$ denotes the class of $\gamma \circ u$ in $\pi_{0}\left(S^{3^{S^{2} \times S^{\prime}}}\right)$ [9].

We note that it is immaterial whether the "top" or "bottom" row map is used to define the index. For

$$
\left(\begin{array}{ll}
\alpha & \beta \\
\gamma & \delta
\end{array}\right)=\left(\begin{array}{ll}
0 & 1 \\
1 & 0
\end{array}\right)\left(\begin{array}{cc}
\gamma & \delta \\
\alpha & \beta
\end{array}\right)
$$

and $U=\left(\begin{array}{ll}0 & 1 \\ 1 & 0\end{array}\right)$ is unitary. If we write $U=e^{i H}$ for some Hermitian $H$, then $U_{t}=e^{i t H}$ is a homotopy between $U$ and the identity.

THEOREM 10. For the algebra $\mathfrak{Q}=M_{2}^{S^{2} \times S^{1}}$, where $\mathcal{G}=\mathrm{GL}_{2}^{S^{2} \times S^{1}}$ and for the index $i$ described above, $\mathcal{H} \neq \mathcal{G}$.

Proof. We shall describe a class of elements contained in $\mathcal{H}-\mathcal{G}$. Consider those elements in $M_{2}^{S^{2} \times S^{1}}$ of the form

$$
h(x)=\left(\begin{array}{ll}
\alpha(x) & \overline{\beta(x)} \\
\beta(x) & \tau(x)
\end{array}\right)
$$


where $\alpha(x) \geqslant m>0, \tau(x) \geqslant|\beta(x)|$ and $m>3\|\tau\|_{\infty}$. If $\tau(x)=0$ on $S^{2} \times$ $S^{1}$, then $h \notin \mathcal{G}$. On the other hand, $h(x) \geqslant 0$ so that $h \in \overline{\mathcal{G}}$. Now if $\|p u-h\|<\varepsilon$ for

$$
p=\left(\begin{array}{ll}
p_{1} & \bar{p}_{2} \\
p_{2} & p_{3}
\end{array}\right) \quad \text { positive }
$$

and

$$
u^{*}=\left(\begin{array}{ll}
u_{1} & u_{2} \\
u_{3} & u_{4}
\end{array}\right) \quad \text { unitary }
$$

then

$$
\left|p_{3}\right|<\left|p_{3}-\left(\bar{\beta} u_{1}+\tau u_{3}\right)\right|+|\beta|+|\tau|<\varepsilon+\|\tau\|_{\infty}
$$

Hence,

$$
\left|\alpha u_{2}\right| \leqslant\left|\left(\bar{p}_{3}-\beta u_{4}\right)-\alpha u_{2}\right|+\left|p_{3}-\bar{\beta} u_{4}\right|<2 \varepsilon+\|\tau\|_{\infty} .
$$

It follows that

$$
\left\|u_{2}\right\|_{\infty} \leqslant\left\|\alpha^{-1}\right\|_{\infty} \varepsilon+\left\|\alpha^{-1}\right\|_{\infty}\|\tau\|_{\infty}<(6 \varepsilon+m) / 3 m .
$$

If $\varepsilon>0$ is chosen so that $\varepsilon<m / 3$, then $\|u\|_{\infty}<1$. This implies that the range of $\gamma \circ u^{*}$ is not all of $S^{3}$. Therefore, $i\left(u^{*}\right)=-i(u)=\left[\gamma \circ u^{*}\right]=0$, since $\gamma$ is a homeomorphism. Hence if $\varepsilon<m / 3, h$ is an element of $\mathcal{H}_{0}-\mathcal{G}_{0}$.

It follows from Proposition 1 that $\mathfrak{H}_{r}-\xi_{r}$ is nonempty for all $r$.

COROLlary 11. There exists a continuous extension of the analytic index on

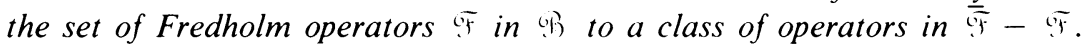

Proof. The analytic index $i_{\text {an }}$ on $\mathcal{F}$ is given by $\sigma \circ i$, where $\sigma$ is the symbol map and $i$ is the topological index on $\mathrm{GL}_{2}^{S^{2} \times S^{1}}$. Since $\sigma$ has a continuous cross section, $i_{\text {an }}$ can be continuously extended to those operators in $\overline{\mathscr{F}}-\widetilde{F}$ whose symbols lie in $\mathcal{H}$.

\section{REFERENCES}

1. F. V. Atkinson, The normal solubility of linear equations in normed spaces, Mat. Sb. 28(70)(1951), 3-14. (Russian) MR 13, 46.

2. L. A. Coburn, The $C^{*}$-algebra generated by an isometry. II, Trans. Amer. Math. Soc. 137(1969), 211-217. MR 38 \#5015.

3. L. A. Coburn and A. Lebow, Algebraic theory of Fredholm operators, J. Math. Mech. 15(1966), 577-584. MR 33 \#569.

4. H. O. Cordes, The algebra of singular integral operators in $R^{n}$, J. Math. Mech. 14(1965), 1007-1032. MR 31 \#6137.

5. R. G. Douglas, Banach algebra techniques in the theory of Toeplitz operators, CBMS Regional Conf. Series in Math., no. 15, Amer. Math. Soc., Providence, R.I., 1973.

6. J. Feldman and R. V. Kadison, The closure of the regular operators in a ring of operators, Proc. Amer. Math. Soc. 5(1954), 909-916. MR 16, 935.

7. M. Gartenberg, Extensions of the index in factors of type $\Pi_{\infty}$, Proc. Amer. Math. Soc. 43(1974), 163-168. MR 48 \#6956.

8. H. L. Royden, Function algebras, Bull. Amer. Math. Soc. 69(1963), 281-298. MR 26 \#6817.

9. R. T. Seeley, The index of elliptic systems of singular integral operators, J. Math. Anal. Appl. 7(1963), 289-309. MR 28 \#2464.

Department of Mathematics, Bernard M. Baruch College, City University of New YORK, NEW YORK, NeW YORK 10010 